\title{
Endothelial Dysfunction in Mexican Obese Children, is there A Role of the Gut Microbiota?
}

\author{
Khemlal Nirmalkar ${ }^{1,2}$, Selvasankar Murugesan ${ }^{1}$, María Luisa Pizano-Zárate ${ }^{3}$, María del \\ Socorro Romero-Figueroa ${ }^{4}$, Carlos Hoyo-Vadillo² ${ }^{2}$ and Jaime Garcia-Mena ${ }^{1 *}$ \\ ${ }^{1}$ Department of Genetics and Molecular Biology, Center for Research and Advanced Studies of the National \\ Polytechnic Institute. Av IPN 2508 Col Zacatenco, Mexico City, 07360 \\ ${ }^{2}$ Department of Pharmacology, Center for Research and Advanced Studies of the National Polytechnic Institute. Av \\ IPN 2508 Col Zacatenco, Mexico City, 07360 \\ ${ }^{3}$ Department of Nutrition, National Institute of Perinatology, Mexico \\ ${ }^{4}$ Coordination of Health Research, Delegation Head of Medical Services IMSS Toluca \\ ${ }^{\#}$ Authors contributed equally to this article
}

Received: : December 01, 2016; Accepted: December 24, 2016; Published: January 04 , 2017

*Corresponding author: Jaime Garcia-Mena, Department of Genetics and Molecular Biology, Center for Research and Advanced Studies of the National Polytechnic Institute. Av IPN 2508 Col Zacatenco, Mexico City, 07360; E-mail: jgmena@cinvestav.mx

\begin{abstract}
Endothelial dysfunction is a predisposing factor for atherosclerosis and other cardiovascular diseases; and at the same time, endothelial dysfunction is associated to obesity. The prevalence of endothelial dysfunction, atherosclerosis and obesity is quite high among Mexican children; on the other hand, gut microbiota has been associated to obesity in the same population. Atherosclerosis is associated to gut microbiota in humans, and this association raise the possibility that endothelial dysfunction is also related with the gut microbiota in obese population. This review addresses the current knowledge of association of endothelial dysfunction, with obesity and atherosclerosis; however, there are no reports of association of endothelial dysfunction with gut microbiota through the measurement of adhesion molecules like ICAM-1, VCAM-1, and selectins.
\end{abstract}

Keywords: Microbiota; Obesity; Endothelial Dysfunction; Children; SCFA

\section{Endothelial Dysfunction}

Endothelium is the thin layer of simple squamous cells that lines the interior surface of blood vessels and lymphatic vessels, forming an interface between circulating blood or lymph in the lumen and the rest of the vessel wall. Endothelial Dysfunction (EDF) is a state of imbalance of vasodilatation or vasoconstriction, or diminished availability of (NO) Nitric oxide [Figure 1]. Here NO works as a vasodilator. This imbalance leads down-regulation of endothelial NO Synthase (eNOS), up-regulation of Reactive Oxygen Species (ROS) [1], C - Reactive Protein (CRP) [2], Vascular Cell Adhesion Molecule-1 (VCAM-1) [3] and it further causes loss of endothelial dependent dilation, blood thickening and formation of small plates. In addition, this imbalance causes impairment of endothelium- dependent vasodilatation and it may cause further atherosclerosis and other cardiovascular diseases [4]. Endothelial dysfunction can be diagnosed by the gold standard method - angiography with acetylcholine injection, with Flow Mediated Dilation (FMD) method, or by measurement of endothelial dysfunction markers - VCAM-1, Intercellular Adhesion Molecule-1 (ICAM-1), E-selection and CRP in blood $[5,6]$.

\section{Endothelial Dysfunction and Atherosclerosis}

Atherosclerosis is a hardening process of the arteries, in which plaque builds up inside the arteries. Plaque is made of cholesterol, fatty substances, cellular waste products, calcium and fibrin. It can block the blood's flow through an artery in the heart and that may lead a heart attack or stroke (American Heart Association, 2014). Endothelial dysfunction is an early marker for atherosclerosis. In Mexico, around 29.8\% children are suffering with endothelial dysfunction [7]. It has been reported that atherosclerosis was observed in 53\% in Mexico during 2005-2007.

\section{Obesity}

Obesity is a metabolic disease and a worldwide health issue. In 2014, more than 600 million adults were obese and 41 million

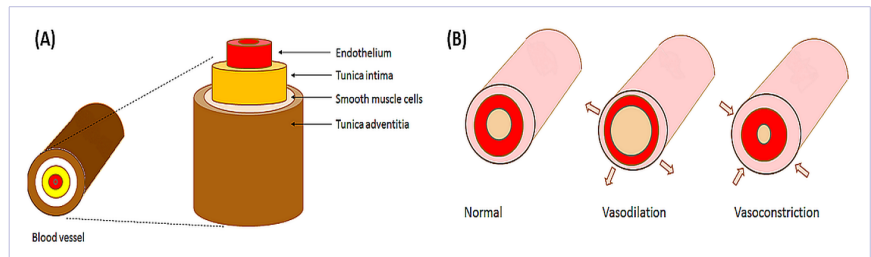

Figure 1: (a) Structure of a blood vessel showing the endothelium, (b) Normal function of a blood vessel 
children under the age of 5 were overweight or obese in 2014 [8]. Initially, obesity was restricted to some developed country but now it has become a global health problem and the prevalence of obesity has also increased from few decades [9]. Obesity and overweight are the fifth death cause with more than 3 million deaths worldwide [10]. In Mexico, more than $70 \%$ adults are overweight [11], 13.3\% of children between the age of 5-11 years old are obese [12], and 1 out of 3 children are overweight [13].

\section{Gut Microbiota}

The development of obesity has influence of human gut microbiome [14]. A human gut is occupied by up to -100 trillion of microorganisms [15], these gut microorganisms help us to balance the digestive system. Alterations in gut microbiome are associated with obesity and with weight loss [16]. Human gut microbial population is dominated by five bacterial phyla; Firmicutes, Bacteroidetes, Actinobacteria, Proteobacteria and Verrucomicrobia. On the other hand, obesity is associated with changes in the relative abundance of phylum Firmicutes and Bacteroidetes [15]. Recent studies showed that the relative abundance of phylum Firmicutes was higher than Bacteroidetes in a sample of United States obese population [17]. In addition, it was reported that bacterial genera like Roseburia spp., Blautia spp., Coprococcus spp., Faecalibacterium spp. and families like Lachnospiraceae, Enterobacteriaceae relative abundance are significantly higher in Mexican overweight and obese children than normal children [18]. These bacteria are associated with high capacity of energy harvest in obese population [19]. Anaerobic intestinal gut microbiota ferments the dietary fiber, and produces Short-Chain Fatty Acids (SCFAs) such as acetic acids, propionic acids and butyric acid [20] and regulates the host energy metabolisms $[21,22]$. In animal models, it has been found that genetically obese $o b / o b$ mice shows high amount of SCFAs in their caecum and less in their feces as comparative to their lean littermates [19]. These SCFAs have many positively effect on intestinal health such as, an energy source for the colonic epithelium (butyrate), and for peripheral tissues (acetate and propionate), an inflammation modulator, helps in the gut motility and in wound healing, nurture the blood flow by vasodilating the colonic vessels as well. In addition, propionate augments the muscular activity, epithelial cell proliferation, and butyrate as a main energy source for colonocytes [21].

\section{Diet Alters the Gut Microbiota}

Diet plays an important role in human health by modulating the gut microbial composition. High fat diet is associated with obesity whereas dietary fiber-rich diet can reduce the risk of obesity. It was suggested that obese microbiota might produce more SCFAs and extract more energy from provided diet than lean microbiota [18]. A dietary fiber containing food enriches the gut microbiota and protects the colonic mucus barrier and it reduces the risk of colitis. Whereas, dietary fiber-free diet increases the mucus-eroding microbiota which degrades the colonic mucus barrier and allow mucosal pathogens like Citrobacter rodentium bacteria to epithelial layer which can cause colitis [23]. A recent study showed that dietary fiber-free diet and captivity increases the intestinal colonization of bacteria Bacteroides spp. and Prevotella spp. in Non Human Primates (NHPs), which reflects in parallel the converge of NHPs microbiota towards the modern human microbiota, and suggests the recent loss of core microbiota in humans [24]. These bacterial genera Bacteroides spp., Prevotella spp. or Ruminococcus spp. are dominant in each enterotype and are associated with the consumption of a diet and not affected by gender, age or nationality [25].

\section{Overview of a Molecular Mechanism Associating Endothelial Dysfunction, Atherosclerosis and Obesity}

Atherosclerosis can cause by any stress to the endothelium like aging, systemic arterial high blood pressure, hypercholesterolemia, Diabetes, smoking, hypertension and with obesity as well. Any of these factors can damage the endothelium and stimulate the secretion of primary proinflammatory cytokines, such as Interleukin-1 (IL-1) and the Tumor Necrosis Factor- $\alpha$ (TNF- $\alpha$ ). These cytokines enhance the expression of adhesion molecules such ICAM-1, VCAM-1 and P-, E-, and L-selectins [Figure 2].

Obese children between the ages 5 to 17 years have more risk of cardiovascular disease. Obesity is associated of high levels of cholesterol, triglycerides in adipose tissues which induces many proinflammatory molecules such as TNF- $\alpha$, IL-6, leptin, Plasminogen Activator Inhibitor-1 (PAI-1), angiotensin-II, resistin and CRP, Monocytes Chemoattractant Protein-1 (MCP-1). These molecules can cause vascular inflammations. Obese also associated with high levels plasma-CRP which directly reflects the high body fat [26]. Angiotensin-II can stimulate the production of ROS which can oxidize the Low-Density Lipoprotein (LDL). These high-levels of CRP, ROS and oxidized Low-Density Lipoprotein (oxLDL) reduce the availability of intracellular nitric oxide $[1,2]$. In addition, they increase the expression of adhesion molecules as well. These adhesion molecules - VCAM-1, ICAM-1 and E-selectins helps the adhesion of blood leukocytes such as monocytes, T-cells, dendritic cells and mast cells to the endothelium layer. Furthermore, leukocytes trans-migrate into inner layer of endothelium called tunica intima where monocytes convert into macrophages; along with remaining leukocytes participates in inflammatory immune reactions that's make plaques. These reactions lead impairment of endothelial function, which later can appear as atherosclerosis [27].

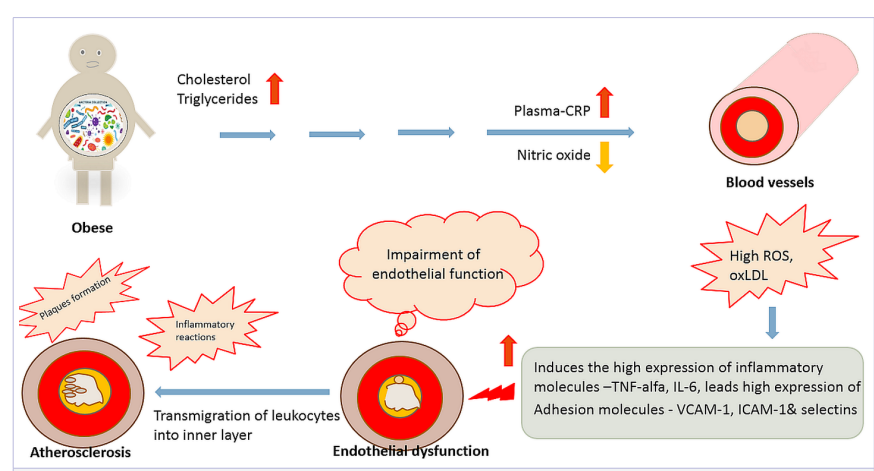

Figure 2: Overview of a molecular mechanism associating with endothelial dysfunction, atherosclerosis and obesity 


\section{Endothelial Dysfunction, Obesity and Gut Microbiota}

Atherosclerosis and obesity are associated with endothelial dysfunction and obesity with gut microbiota [17]. Recent studies suggested that the gut metagenome is associated with the symptomatic atherosclerosis [28]. As we know atherosclerosis is a deposition of lipid molecules and the gut microbiota causes inflammation by Lipopolysaccharide (LPS) and peptidoglycan that can lead metabolic disease. Pyrosequencing showed that atherosclerotic plaque contains bacterial DNA [29]. Shotgun sequencing of the gut metagenome revealed that the genus Collinsella spp. was dominated in patients with symptomatic atherosclerosis whereas Roseburia spp. and Eubacterium spp. were observed more in healthy controls [28].

Obese children between the ages 5 to 17 years have more risk of cardiovascular disease. Recently, our group has reported the microbial diversity in Mexican obese children [18]. We found that the relative abundance of the phylum Firmicutes was more than the Bacteroidetes in obese children with high-level triglycerides and cholesterol. We also observed the SCFAs concentration in feces was lower in obese children feces than normal weight; it was attributed to an increase in the mucosal absorption of SCFAs. EDF and atherosclerosis prevalence is slightly higher in Mexican population. Although, there is no report up to our knowledge about association between EDF and gut Microbiota, even in the Mexican population. However, we believe SCFAs and gut microbiota play a role in endothelial dysfunction by inflammatory molecules in overweight and obese Mexican children population [Figure 3].

\section{Conclusion}

Endothelial dysfunction is a predisposing factor for atherosclerosis and for other cardiovascular diseases, and although there is a solid association of the gut microbiota with obesity in Mexican children, it is necessary to investigate the association between EDF and the gut microbial diversity or function. Further studies are necessary to establish whether a consequence of EDF is to alter the gut microbiota composition or its function. A more appealing hypothesis is that a primary gradual change occurs in the microbiota attributes before the appearance of EDF in children. An altered gut microbiota could contribute significantly to endothelial function by the production of currently undescribed bioactive metabolites.

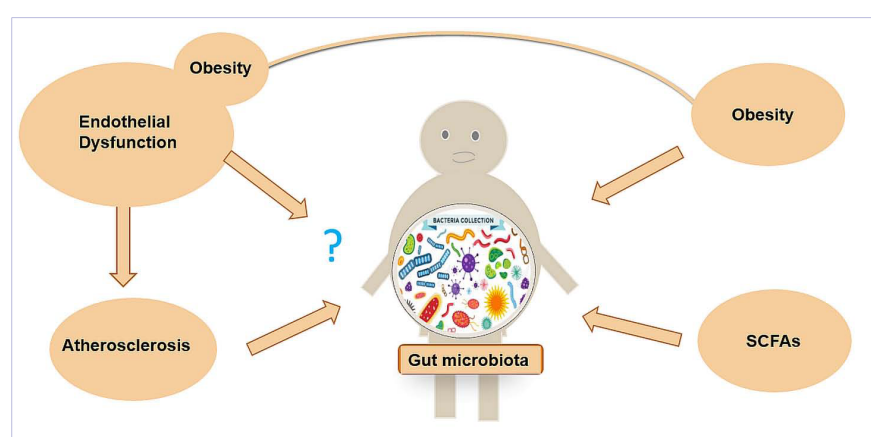

Figure 3: Association of gut microbiota with different factors (see text)

\section{Acknowledgement}

This work was funded by Cinvestav; CONACyT 163235 INFR-2011-01; and FONSEC SS/IMSS/ISSSTE-CONACYT-233361 granted to JGM. We thank a Postdoctoral Fellowship from FONSEC SS/IMSS/ISSSTE-CONACYT-233361 granted to SM.

\section{References}

1. Cominacini L, Rigoni A, Pasini AF, Garbin U, Davoli A, Campagnola M, et al. The binding of oxidized low-density lipoprotein (ox-LDL) to oxLDL receptor reduces the intracellular concentration of nitric oxide in endothelial cells through an increased production of superoxide. J Biol Chem. 2001;276(17):13750-5.

2. Verma S, Wang CH, Li SH, Dumont AS, Fedak PW, Badiwala MV, et al. A self-fulfilling prophecy: C-reactive protein attenuates nitric oxide production and inhibits angiogenesis. Circulation. 2002;106(8):913-9.

3. Khan BV, Harrison DG, Olbrych MT, Alexander RW, Medford RM. Nitric oxide regulates vascular cell adhesion molecule 1 gene expression and redox-sensitive transcriptional events in human vascular endothelial cells. Proc Natl Acad Sci U S A, 1996;93(17):9114-9.

4. Hadi ARH, Cornelia SC, Suwaidi JA. Endothelial dysfunction: cardiovascular risk factors, therapy, and outcome. Vasc Health Risk Manag. 2005;1(3):183-198.

5. Lopez-Garcia E, Schulze MB, Fung TT, Meigs JB, Rifai N, Manson JE, et al. Major dietary patterns are related to plasma concentrations of markers of inflammation and endothelial dysfunction. Am J Clin Nutr. 2004;80(4):1029-35.

6. Eikemo H, Sellevold OF, Videm V. Markers for Endothelial Activation during Open Heart Surgery. Ann Thorac Surg. 2004;77(1):214-9.

7. Carranza MJ, López CSM, García GV, López RT, Botello GG, Campos PY. Valores de función endotelial en niños mexicanos. Med Int Mex 2011;27(5):429-438.

8. Obesity and overweight prevalence worldwide, WHO 2016. Available online at http://www.who.int/mediacentre/factsheets/fs311/en/

9. Ng M, Fleming T, Robinson M, Thomson B, Graetz N, Margono C, et al. Global, regional, and national prevalence of overweight and obesity in children and adults during 1980-2013: a systematic analysis for the global burden of disease study 2013. Lancet. 2014;384(9945):766-81. doi: 10.1016/S0140-6736(14)60460-8.

10. World Health Organization (WHO) (2008).The global burden of disease: 2004 update. WHO, Geneva, Switzerland.

11. National Health survey, OCED, Mexico, 2014. Available on at http:// www.oecd.org/mexico/Obesity-Update-2014-MEXICO_EN.pdf

12. Encuesta Nacional de Salud y Nutrición. Resultados Nacionales 2012. Instituto Nacional de Salud Pública. Available online at: http:// ensanut.insp.mx/informes/ ENSANUT2012ResultadosNacionales.pdf

13. The International association for the study of obesity, 2013. Available online at http://www.oecd.org/mexico/Obesity-Update-2014MEXICO_EN.pdf

14. Devaraj S, Hemarajata P, Versalovic J. The human gut microbiome and body metabolism: implications for obesity and diabetes. Clin Chem. 2013;59(4):617-28. doi: 10.1373/clinchem.2012.187617.

15. Ley RE1, Bäckhed F, Turnbaugh P, Lozupone CA, Knight RD, Gordon JI. Obesity alters gut microbial ecology. Proc Natl Acad Sci U S A. 2005;102(31):11070-5. 
16. Ley RE. Obesity and the human microbiome. Curr Opin Gastroenterol. 2010;26(1):5-11. doi: 10.1097/MOG.0b013e328333d751.

17. Ley RE, Turnbaugh P, Klein S, Gordon J. Microbial ecology: human gut microbes associated with obesity. Nature. 2006; 444:1022-1023.

18. Murugesan S, Ulloa-Martínez M, Martínez-Rojano H, Galván-Rodríguez FM, Miranda-Brito C, Romano MC, et al. Study of the diversity and short-chain fatty acids production by the bacterial community in overweight and obese Mexican children. Eur J Clin Microbiol Infect Dis. 2015;34(7):1337-46. doi: 10.1007/s10096-015-2355-4.

19. Turnbaugh PJ, Ley RE, Mahowald MA, Magrini V, Mardis ER, Gordon JI. An obesity-associated gut microbiome with increased capacity for energy harvest. Nature. 2006;444(7122):1027-1031.

20. Gill SR1, Pop M, Deboy RT, Eckburg PB, Turnbaugh PJ, Samuel BS, et al. Metagenomic analysis of the human distal gut microbiome. Science. 2006;312(5778):1355-1359.

21. Topping DL. Short-chain fatty acids produced by intestinal bacteria Asia Pac J Clin Nutr. 1996;5(1):15-9.

22. den Besten G, van Eunen K, Groen KA, Venema K, Reijngoud JD, Bakker MB. The role of short-chain fatty acids in the interplay between diet, gut microbiota, and host energy Metabolism. J Lipid Res. 2013;54(9):2325-40. doi: 10.1194/jlr.R036012.
23. Desai MS, Seekatz AM, Koropatkin NM, Kamada N, Hickey CA, Wolter $M$, et al. A Dietary Fiber-Deprived Gut Microbiota Degrades the Colonic Mucus Barrier and Enhances Pathogen Susceptibility. Cell. 2016;167(5):1339-1353.e21. doi: 10.1016/j.cell.2016.10.043.

24. Clayton JB, Vangay P, Huang H, et al. Captivity humanizes the primate microbiome. Proc Natl Acad Sci USA. 2016;113(37):10376-81.

25. Arumugam M, Raes J, Pelletier E, Le Paslier D, Yamada T, Mende DR, et al. Enterotypes of the human gut microbiome. Nature. 2011;473(7346):174-80. doi: 10.1038/nature09944.

26. Pasceri V, Willerson JT, Yeh ET. Direct proinflammatory effect of C-reactive protein on human endothelial cells. Circulation. 2000;102(18):2165-8.

27. Libby P.Inflammation in atherosclerosis. Nature. 2002;420(6917):86874.

28. Karlsson FH, Fåk F, Nookaew I, Tremaroli V, Fagerberg B, Petranovic D, et al. Symptomatic atherosclerosis is associated with an altered gut metagenome. Nat Commun. 2012;3:1245. doi: 10.1038/ncomms2266.

29. Koren 0, Spor A, Felin J, Fåk F, Stombaugh J, Tremaroli V, et al. Human oral, gut, and plaque microbiota in patients with atherosclerosis. Proc Natl Acad Sci U S A. 2011;108 Suppl 1:4592-8. doi: 10.1073/ pnas.1011383107. 